\title{
O impacto da histerectomia total sobre a função sexual feminina
}

\author{
The impact of hysterectomy on female sexual function
}

\author{
El impacto de la histerectomía en la función sexual femenina
}

Antonina Beatriz da Costa Rodrigues ${ }^{1}$, Lucelayne Crislly Costa de Almeida ${ }^{1}$, Rafaella Carolina Brito da Cruz $^{1}$, Elaine Glauce Santos de Souza ${ }^{1 *}$, Hellen Vivianni Veloso Corrêa ${ }^{2}$, Susanne Cristine Brito e Silva ${ }^{1}$.

\section{RESUMO}

Objetivo: Analisar o impacto da histerectomia total sobre a função sexual feminina e sua relação com sintomas depressivos. Métodos: Trata-se de um estudo observacional transversal, de análise quantitativa, através da aplicação de questionários. Foram aplicados 3 questionários em uma amostra de 42 mulheres, cuja idade era entre 27 e 65 anos e que tinham no mínimo 2 meses de pós-operatório de histerectomia total. Resultados: Os dados sobre a função sexual e a presença de sintomas depressivos foram verificados pelos Questionários de Female Sexual Function Index (FSFI) e o Inventário de Depressão de Beck. A correlação entre as variáveis acima foi verificada através de análises estatísticas e ilustradas em forma de tabelas estratificadas por escores total do FSFI, escore total de Beck, escore dos domínios e sua relação com os sintomas depressivos. Conclusão: Os resultados encontrados foram significativos e mostraram que a diminuição na função sexual pode levá-las a apresentar sintomas depressivos.

Palavras-chave: Histerectomia, Útero, Sexualidade, Mulheres.

\begin{abstract}
Objective: To analyze the impact of total hysterectomy on female sexual function and its relationship with depressive symptoms. Methods: This was a cross-sectional observational study, with quantitative analysis, through the application of questionnaires. Three questionnaires were applied to a sample of 42 women, whose age was between 27 and 65 years old and who had at least 2 months after the total hysterectomy. Results: The data on sexual function and the presence of depressive symptoms were verified by the Female Sexual Function Index Questionnaires (FSFI) and the Beck Depression Inventory. The correlation between the variables above was verified through statistical analysis and illustrated in the form of tables stratified by total FSFI scores, total Beck score, domain score and its relationship with depressive symptoms. Conclusion: It is concluded that the results found were significant and showed that the decrease in sexual function can lead to depressive symptoms.
\end{abstract}

Keywords: Hysterectomy, Uterus, Sexuality, Women.

\section{RESUMEN}

Objetivo: Analizar el impacto de la histerectomía total en la función sexual femenina y su relación con los síntomas depresivos. Métodos: Se trata de un estudio observacional transversal, con análisis cuantitativo, mediante la aplicación de cuestionarios. Se aplicaron tres cuestionarios a una muestra de 42 mujeres, cuya edad estaba entre 27 y 65 años y que tenían al menos 2 meses después de la histerectomía total. Resultados: Los datos sobre la función sexual y la presencia de síntomas depresivos fueron verificados por los

1 Universidade da Amazônia (UNAMA), Belém - PA. *E-mail: elaineglaucesouza@hotmail.com

2 Universidade Federal do Pará (UFPA), Belém - PA.

SUBMETIDO EM: 5/2021

PUBLICADO EM: 5/2021 
Cuestionarios del Índice de Función Sexual Femenina (FSFI) y el Inventario de Depresión de Beck. La correlación entre las variables anteriores se verificó mediante análisis estadístico y se ilustró en forma de tablas estratificadas por puntajes totales de FSFI, puntaje total de Beck, puntaje de dominio y su relación con los síntomas depresivos. Conclusión: Los resultados encontrados fueron significativos y mostraron que la disminución de la función sexual puede conducir a síntomas depresivos.

Palabras clave: Histerectomía, Útero, Sexualidad, Mujeres.

\section{INTRODUÇÃO}

O útero é um órgão pélvico, cujo formato se assemelha a uma pera e é importante para o ciclo reprodutivo dos seres humanos. Por outro, as funções biológicas do útero, estão associadas ao conceito de feminilidade por relacionarem-se ao papel reprodutor da mulher, bem como a sua vida sexual. (ADORNO SS, et al., 2018; TOZO IM, et al., 2009). Entretanto, em decorrência a algumas enfermidades que acometem esta região, tais como doenças ginecológicas benignas, câncer de colo uterino, prolapso uterino, mioma uterino e para o tratamento de sangramento disfuncional, a histerectomia é a intervenção cirúrgica mais indicada (AARTS JWM, et al., 2015).

A histerectomia é um procedimento cirúrgico ginecológico no qual realiza-se a remoção do útero, podendo ser efetuada através da via abdominal (principal via utilizada), vaginal ou vídeolaparoscópica. É um dos procedimentos mais frequente em mulheres, ficando em terceiro lugar na frequência entre as principais operações de cirurgia eletiva (CARDOSO BC, et al., 2017; COELHO SM, 2015).

Existem diversos tipos de histerectomia, onde todas envolvem a remoção do corpo uterino, também podendo compreender a remoção das trompas de falópio (salpingectomia) e ovário (ooforectomia). Existem também, diversas vias cirúrgicas, dentre elas, a histerectomia subtotal, radical e total (COSTA JC e COSTA $A, 2017)$. A histerectomia total se refere a remoção do corpo e colo do útero e é superada apenas pela cesariana, uma vez que cerca de 20 a $30 \%$ das mulheres serão submetidas a esta operação até a sexta década de vida (COELHO SM, 2015; GHIELMETTI T, et al., 2006).

Algumas pesquisas relatam que as taxas de ocorrência da histerectomia podem ser aumentadas em decorrências de fatores como as características sociodemográficas, do padrão reprodutivo e de práticas contraceptivas como o uso do dispositivo intra-uterino ou de uso de contraceptivos orais por longo prazo. É uma das cirurgias ginecológicas mais realizadas no mundo, sendo considerada a segunda cirurgia ginecológica mais frequentemente realizada em mulheres nos países desenvolvidos. (ARAÚJO TVB e AQUINO EML, 2015).

No Brasil, a cada ano, aproximadamente 300 mil mulheres recebem a indicação para a realização de histerectomia e necessitam desta cirurgia. Segundo dados do Departamento de informática do Sistema Único de Saúde do Brasil (DATASUS), no ano de 2015 foram realizadas no país cerca de 53.753 histerectomias totais pelo Sistema Único de Saúde (SUS) (REAL AA, et al., 2012). Em contrapartida, em alguns países como Israel, Espanha, Portugal, República Checa e Dinamarca, a taxa de realização de histerectomia é menor, cerca de menos de 200 por 100.000 mulheres por ano (COSTA JC e COSTA A, 2017).

Além disso, a histerectomia possui vantagens podendo ser considerada pela mulher como preditora de implicações positivas, que estão relacionadas à ausência de sintomas gerados pela doença o qual obteve-se à indicação e realização da cirurgia (MERIGHI MAB, et al.,2012). Por outro lado, o processo cirúrgico pode acarretar várias alterações no processo de viver da mulher, tais como a perda da possibilidade de reprodução e parada do ciclo menstrual. Como o útero possui sua função biológica e fisiológica ligada à maternidade e representa socialmente a sexualidade, essas complicações poderão interferir negativamente na autoimagem, autoestima e na qualidade de vida das mulheres, intervindo até mesmo na vida conjugal e nas relações sociais destas (NUNES MPRS, et al., 2009).

Desta forma, estudos demostram que mulheres submetidas à histerectomia podem apresentar alterações no autoconceito, sintomas depressivos e psicossomáticos, prejuízos na qualidade da vida sexual, nas 
condições psicológicas e na qualidade do relacionamento estabelecido com o parceiro dificuldade de relacionamento sexual e conjugal. Além disso, em razão as associações psicológicas feitas entre a gestação e a sexualidade, em alguns casos, podem provocar emoções conflitivas, traumáticas, sentimento de insegurança e ansiedade, provocando assim, importantes mudanças nos padrões e no desejo sexuais (CARVALHO HCM e LEMOS MF, 2017; TOZO IM, et al., 2009).

No que se refere aos aspectos emocionais, a realização de uma histerectomia pode desencadear fortes consequências, entre elas está a ansiedade, que é relacionada à perda do útero e ao próprio desconhecimento do corpo feminino e da função dos órgãos envolvidos na excitação e no prazer sexual, podem interferir de forma desfavorável na obtenção de satisfação em ambos os parceiros bem como provocam mudanças consideráveis no comportamento, no desejo sexual e na qualidade de vida da mulher. (MELO MCB e BARROS EM, 2009).

Além dos aspectos emocionais, ocorrem também modificações anatômicas na região da pelve, podendo levar a uma modificação do tamanho e/ou do formato dos órgãos genitais, dificuldade de penetração durante o ato sexual, dispareunia, interrupção da sustentação anatômica da resposta sexual, redução da libido e do grau de atração, uma vez que, ocorre a redução de níveis hormonais circulantes derivados de alterações na circulação, ocasionando, em alguns casos, disfunções sexuais. Além disso, alguns estudos relatam que a histerectomia pode causar encurtamento da vagina e menor frequência de orgasmos após a penetração vaginal (REAL AA, et al., 2012; TOZI IM, et al., 2009).

Desta forma, é de extrema importância explicações e informações claras sobre o procedimento cirúrgico e suas possíveis consequências a fim de aliviar a ansiedade e melhorar a imagem corporal, bem como esclarecimentos quanto à função do útero, da vagina e explicar que a mulher a região do clitóris como área para a satisfação sexual e o orgasmo (MELO MCB e BARROS EM, 2009).

Baseado nessa relação, o estudo teve como objetivo analisar os efeitos do pós-operatório na histerectomia total sobre a função sexual feminina e sua relação com sintomas depressivos.

\section{MÉTODOS}

Devem descrever de forma clara e sem prolixidade as fontes de dados, a população estudada, a amostragem, os critérios de seleção, procedimentos analíticos e questões éticas relacionadas à aprovação do estudo por comitê de ética em pesquisa (pesquisa com seres humanos e animais) ou autorização institucional (levantamento de dados onde não há pesquisa direta com seres humanos ou animais).

Trata-se de um estudo observacional transversal, de análise quantitativa, através da aplicação de três questionários. A pesquisa foi realizada em um Ambulatório da Mulher, de uma cidade do estado do Pará, no período de setembro a novembro de 2019 , em um período de quatro vezes por semana (segunda, terça, quarta e quinta). Os critérios para inclusão foram mulheres entre 27 e 65 anos de idade, ter entre 2 meses a 10 anos de pós-operatório de histerectomia total; estar em acompanhamento médico. Os critérios de exclusão foram: a paciente não ter vida sexual ativa antes da cirurgia ginecológica, está fora do período de tempo estabelecido, está fazendo uso de algum medicamento para depressão, mulheres analfabetas (pois os questionários são auto aplicados), mulheres com alterações cognitivas e que apresentem prolapsos de órgãos pélvicos.

Para a coleta de dados foram utilizados três questionários, sendo o Female Sexual Function Index (FSFI), o Questionário de Inventário de Depressão de Beck e o Questionário Socioeconômico. O Female Sexual Function Index (FSFI), é um breve questionário que pode ser autoaplicado, e tem o objetivo de avaliar a resposta sexual feminina nas seguintes fases: desejo sexual, excitação sexual, lubrificação vaginal, orgasmo, satisfação sexual e dor. Este, possui 19 questões que avaliam essas funções sexuais, cada item tem uma pontuação de 0 a 5 sendo de forma crescente a avaliação das questões, exceto com a questão relacionada a dor que é avaliada de forma invertida. Os escores dos domínios e a escala geral de escores do FSFI são calculados como demonstrado na Tabela 1. 
Tabela 1 - Escores dos domínios do FSFI.

\begin{tabular}{cccccc}
\hline Domínio & Questão & $\begin{array}{c}\text { Variação de } \\
\text { escore }\end{array}$ & Do fator & $\begin{array}{c}\text { Escore } \\
\text { mínimo }\end{array}$ & $\begin{array}{c}\text { Escore } \\
\text { máximo }\end{array}$ \\
\hline Desejo & 1,2 & $1-5$ & 0,6 & 1,2 & 6,0 \\
Excitação & $3,4,5,6$ & $0-5$ & 0,3 & 0 & 6,0 \\
Lubrificação & $7,8,9,10$ & $0-5$ & 0,3 & 0 & 6,0 \\
Orgasmo & $11,12,13$ & $1-5$ & 0,4 & 0 & 6,0 \\
Satisfação & $14,15,16$ & 0 (ou 1) - 5* & 0,4 & 0,8 & 6,0 \\
Dor & $17,18,19$ & $0-5$ & 0,4 & 0 & 6,0 \\
\hline Escore total & \multicolumn{5}{c}{2,0} \\
\hline Legenda: *Variação para o item 14= 0-5; Variação para os itens 15 e 16=1-5. \\
Fonte: Rodrigues ABC, et al., 2021.
\end{tabular}

Para escores dos domínios, somam-se os escores individuais e multiplica-se pelo fator correspondente. Para obter o escore total da escala soma-se os escores para cada domínio, que apresenta valores mínimos de 2 e máximo de 36, sendo os maiores valores associados a uma melhor função sexual. Deve ser observado que dentro dos domínios, um escore zero indica que a paciente relatou não ter tido atividade sexual nas últimas quatro semanas. Esse questionário foi validado por Hentschel T, et al. (2007), sendo que foi aplicada a versão adaptada ao português por Pacagnella RC, et al. (2008).

O Questionário de Inventário de Depressão de Beck, é utilizado para avaliar sintomas de depressão, é um questionário curto e de fácil compreensão, sendo muito simples o procedimento da pontuação. Possui 21 itens e pode ser usado por qualquer pessoa com mais de 13 anos de idade, sua classificação é por uma escala de quatro pontos, sendo de 0 a 3 . 00 indica que a pessoa não apresenta sintomas e o 3 indica uma forma grave de apresentá-los. De acordo com Beck AT, et al. (1988), a escolha do ponto de corte adequado depende da natureza da amostra e dos objetivos do estudo, podendo ser utilizado tanto em amostras com diagnóstico de depressão como em amostras não diagnosticadas para verificar a presença de sintomas depressivos. Para amostras não diagnosticadas, Kendall PC, et al. (1987) recomendam escores acima de 15 para detectar disforia e concluem que o termo "depressão" deve ser apenas utilizado para os indivíduos com escores acima de 20.

O Questionário Socioeconômico é um questionário que possui como objetivo a coleta de informações sobre condições socioeconômicas e culturais, elaborado pelos pesquisadores ao público alvo da pesquisa.

As participantes foram abordadas na sala de espera de atendimento do Ambulatório da Mulher, no horário em que estavam aguardando atendimento médico. No momento da abordagem, os pesquisadores convidaram-nas a participar e fornecendo as devidas informações sobre a pesquisa. As participantes que concordaram em participar do estudo, foram encaminhadas para a sala de acolhimento da enfermagem, onde responderam os questionários, mantendo-se a privacidade das mesmas. Para isto, os pesquisadores entregaram o Termo de Consentimento Livre e Esclarecido (TCLE), e em seguida, foram entregues os questionários Socioeconômico, Questionário FSFI e o questionário de Inventário de Depressão de Beck.

Os questionários são auto aplicados, objetivando avaliar a percepção individual das participantes. Caso houvesse alguma dúvida, os pesquisadores estavam presentes para esclarece-las, sem interferir nas respostas. Depois que as participantes responderam devidamente os questionários, os mesmos foram colocados em um envelope e depois lacrados para evitar o extravio de dados.

A análise estatística foi realizada pelo programa estatístico Statistical Package for the Social Sciences (SPSS) versão 20. Para verificar se havia relação entre sintomas depressivos e o escore total de FSFI, utilizou-se o teste de correlação de Spearman, com nível de significância de $p \leq 0,05$. A estatística descritiva foi feita através do questionário socioeconômico, relação entre escore total do FSFI e escore total de Beck, os domínios do FSFI e o escore de Beck. 


\section{RESULTADOS}

Participaram incialmente do estudo 52 mulheres histerectomizadas, no entanto 10 foram excluídos por não atenderem aos critérios de inclusão. Assim, 42 participantes estão inclusas na pesquisa. Os dados socioeconômicos e gerais estão descritos na Tabela 2 de acordo com as varáveis contidas no Questionário Socioeconômico e Questionário de Inventário de Depressão de Beck conforme preenchido pelas participantes.

Tabela 2 - Descrição dos dados socioeconômicos e gerais.

\begin{tabular}{|c|c|c|c|c|c|}
\hline & Média & $\begin{array}{l}\text { Desvio } \\
\text { padrão }\end{array}$ & Mínimo & Máximo & Porcentagem \\
\hline $\begin{array}{l}\text { Escore de Beck } \\
\qquad(\mathrm{N}=42)\end{array}$ & M: 11,19 & 10,04 & $\begin{array}{l}\text { Escore } \\
\text { mínimo: } 0,0\end{array}$ & $\begin{array}{l}\text { Escore } \\
\text { máximo: } \\
36,00\end{array}$ & $\begin{array}{c}66,7 \% \text { sem presença de } \\
\text { sintomas depressivos } \\
7,1 \% \text { disforia } \\
26,2 \% \text { sintomas } \\
\text { depressivos }\end{array}$ \\
\hline $\begin{array}{l}\text { Idade } \\
(\mathrm{N}=42)\end{array}$ & $\begin{array}{l}\text { M: } 48,35 \\
\text { anos }\end{array}$ & DP: 9,05 & $\begin{array}{l}\text { Idade } \\
\text { mínima: } 27 \\
\text { anos }\end{array}$ & $\begin{array}{l}\text { Idade } \\
\text { máxima: } 65 \\
\text { anos }\end{array}$ & 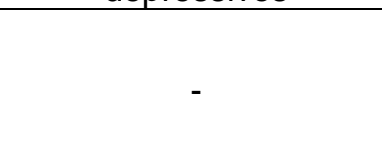 \\
\hline $\begin{array}{l}\text { Escolaridade } \\
\qquad(\mathrm{N}=42)\end{array}$ & - & - & - & - & $\begin{array}{c}28,6 \% \text { ensino } \\
\text { fundamental incompleto } \\
7,1 \% \text { ensino médio } \\
\text { incompleto } \\
38,1 \% \text { ensino médio } \\
\text { completo } \\
7,1 \% \text { ensino superior } \\
\text { incompleto } \\
19 \% \text { ensino superior } \\
\text { completo }\end{array}$ \\
\hline $\begin{array}{c}\text { Tempo de } \\
\text { histerectomia } \\
(\mathrm{N}=42)\end{array}$ & M: 5,86 & DP: 3,42 & $\begin{array}{l}\text { Tempo } \\
\text { mínimo: } 3 \\
\text { meses }\end{array}$ & $\begin{array}{l}\text { Tempo } \\
\text { máximo: } 10 \\
\text { anos }\end{array}$ & - \\
\hline $\begin{array}{c}\text { Retorno a } \\
\text { atividade sexual } \\
(\mathrm{N}=42)\end{array}$ & $\begin{array}{l}\mathrm{M}: 3 \\
\text { meses }\end{array}$ & DP: 0,19 & $\begin{array}{l}\text { Tempo } \\
\text { mínimo: } 2 \\
\text { meses }\end{array}$ & $\begin{array}{l}\text { Tempo } \\
\text { máximo: } 1 \\
\text { ano }\end{array}$ & - \\
\hline
\end{tabular}

Fonte: Rodrigues ABC, et al., 2021.

A média total do $\mathrm{FSFI}$ foi de $26,00(\mathrm{~N}=42, \mathrm{DP}=5,30)$, ou seja, um resultado abaixo de 26,55 , indicando a possibilidade de apresentar disfunção sexual. A média de cada domínio da função sexual está representada na Tabela 3.

Tabela 3 - Média dos domínios do FSFI.

\begin{tabular}{ccc}
\hline Domínio & Média & Desvio padrão \\
\hline Desejo & 3,6 & 0,85 \\
Excitação & 3,96 & 0,98 \\
Lubrificação & 4,26 & 1,23 \\
Orgasmo & 4,55 & 1,20 \\
Satisfação & 4,99 & 1,19 \\
Dor & 4,54 & 1,39 \\
\hline Escore total & 26,00 & 5,30 \\
\hline
\end{tabular}

Fonte: Rodrigues ABC, et al., 2021. 
Houve correlação negativa e fraca entre sintomas depressivos e o escore total de FSFI, rs $=-0,458$, $\mathrm{p}=0,002, \mathrm{r} 2=0,209$ ( $\mathrm{N}=42)$. Portanto, quanto maior o índice de sintomas depressivos, menor é o escore total de FSFI. Por extensão, cerca de $21 \%$ da variação no escore total de FSFI está associado a presença de sintomas depressivos, com o restante decorrente de outros fatores.

Conforme a Tabela 4, as mulheres que realizaram histerectomia apresentaram que os domínios mais afetados após a cirurgia e pela presença de sintomas depressivos, são os escores de lubrificação, satisfação e dor onde foi estatisticamente significativa.

Tabela 4- Domínios afetados pela presença de sintomas depressivos.

\begin{tabular}{ccc}
\hline & Rs & P \\
\hline Domínio lubrificação $(\mathrm{N}=42)$ & $-0,444$ & 0,003 \\
Domínio satisfação $(\mathrm{N}=42)$ & $-0,467$ & 0,002 \\
Domínio dor $(\mathrm{N}=42)$ & $-0,429$ & 0,005 \\
\hline
\end{tabular}

Fonte: Rodrigues ABC, et al., 2021.

\section{DISCUSSÃO}

A histerectomia $O$ objetivo desta pesquisa foi analisar os efeitos do pós-operatório na histerectomia total sobre a função sexual feminina e sua relação com os sintomas depressivos. Os resultados apresentados indicam que a média do FSFI encontrado por essa pesquisa foi de 26,00 , o que indica provável disfunção sexual, segundo Wiegel M (2005), citando que a nota de corte para o Questionário FSFI é abaixo de 26,55.

O resultado desse presente estudo corrobora com os resultados do trabalho de Faleiros NPA (2011), que realizou uma pesquisa com uma amostra de 11 mulheres no pré e pós-operatório de histerectomia, onde foi realizada uma avalição três meses antes e três meses depois da cirurgia, cujo resultado mostrou que 6 participantes apresentaram escores menor ou igual a 26 pontos.

Segundo Tozi IM, et al. (2009), a histerectomia ocasiona modificações na anatomia pélvica, alterando o tamanho e formato dos órgãos genitais, dificuldade de penetração vaginal, dores durante as relações sexuais, interrupção dos suportes anatômicos da resposta sexual, diminuição do desejo sexual e do grau de atratividade, devido à redução de níveis hormonais circulantes, decorrentes de alterações circulatórias, e consequentemente disfunções sexuais.

De acordo com os dados apresentados nesse estudo, os domínios mais afetados após a cirurgia são os referentes à desejo e excitação que estão relacionados com a diminuição de níveis hormonais circulantes como a progesterona e o estrogênio, produzidos principalmente pelos ovários, e os que apresentaram melhores resultados são os domínios de satisfação e orgasmo, visto que envolve conceitos multifatoriais relacionados a qualidade de vida com o parceiro, convívio social, autoconceito e boa auto estima (TOZO IM, et al., 2009).

Segundo o estudo de Santos JLC, et al. (2017), o procedimento de histerectomia pode não ter influência no comprometimento da satisfação sexual melhorando a qualidade de vida após a cirurgia. Já o estudo de Lunelli BP, et al. (2014) avaliou a função sexual em 39 mulheres histerectomizadas e realizou aplicação do questionário que avalia a função sexual, o QS-F, no período pré e pós cirúrgico de 6 meses, os resultados mostram que houve melhora na satisfação sexual. O resultado discorda do presente estudo, visto que não houve avaliação sexual com o questionário de FSFI, nem correlação com sintomas depressivos. Portanto, a literatura refere múltiplos fatores que podem contribuir para o estudo complexo de função sexual após a histerectomia, tendo em vista que os resultados controversos dependem da correlação de fatores biopsicossociais e a prevalência de disfunção sexual feminina varia amplamente, principalmente devido às diferenças metodológicas da cirurgia (ILLIANO E, et al., 2006).

O presente estudo teve um escore médio de sintomas depressivos de 11,19 em uma amostra de 42 mulheres histerectomizadas, de forma que, $7,1 \%$ estão classificados como disforia e $26,2 \%$ como possível 
diagnóstico para depressão, e o restante $(66,7 \%)$ não apresenta indicativos de sintomas depressivos. Esse resultado é semelhante aos resultados encontrados por Bahri $\mathrm{N}$, et al. (2016) que realizaram um estudo longitudinal no intuito de avaliar a presença de sintomas depressivos no período pré e pós cirúrgico de 3 meses em 53 mulheres, no qual não houve relação entre a histerectomia e a presença de sintomas depressivos, cuja média antes da cirurgia era de 13,01 e após a cirurgia era de 11,02.

A histerectomia total pode provocar importantes alterações nos aspectos emocionais, psíquicos, anatômicos e sociais interferindo na qualidade de vida de mulheres histerectomizadas (LUNELLI BP, et al., 2014). A realização de uma histerectomia pode desencadear fortes reações emocionais como ansiedades relacionadas à perda do útero e o próprio desconhecimento do corpo feminino (MELO MCB e BARROS EM, 2009). Na alteração psíquica para algumas mulheres, a histerectomia foi aceita com certa facilidade, pois representava para elas a solução de problemas, especialmente o fim dos sintomas físicos desagradáveis, chegando a ser considerado motivo de alívio (SILVA CMC, et al., 2010).

Em um estudo realizado por Harnod T, et al. (2018), os autores descobriram um risco de 1,02 por 100 pessoas para desenvolver depressão entre mulheres que foram submetidas a uma histerectomia entre $30 \mathrm{e}$ 49 anos. Nos resultados desse estudo, a terapia hormonal não teve efeito significativo na redução da depressão, após uma histerectomia.

De acordo com o resultado do presente estudo, a presença de sintomas depressivos interfere negativamente na vida sexual de mulheres histerectomizadas, indicado pela correlação entre sintomas depressivos e índice geral de FSFI, favorecendo assim um possível surgimento de disfunção sexual.

Segundo Harnod T, et al. (2018), em um estudo de caráter observacional, demonstraram que a ansiedade interagiu com a histerectomia para o desenvolvimento de depressão e ansiedade pós-histerectomia, estresse, mudança na identidade das mulheres e senso de feminilidade, teve um pior desempenho na função sexual e sintomas ginecológicos subjetivos após a cirurgia que podem interagir juntos para afetar essa dimensão importante da vida das mulheres e levar à depressão após a histerectomia. Os resultados deste estudo revelaram que a histerectomia, ooforectomia e uso de hormônio pós-cirúrgico estão associados a um risco aumentado de depressão quando ocorrem sozinhos, mas conferem um risco maior se forem experimentados em conjunto. Esse estudo confirma o presente estudo, visto que a histerectomia pode ocasionar sintomas depressivos afetando a função sexual e qualidade de vida de mulheres histerectomizadas.

Foi encontrado uma correlação negativa entre os domínios lubrificação, satisfação e dor do Questionário FSFI com os sintomas depressivos. Um estudo relatou que a presença de falta de lubrificação vaginal é o fator mais constante relatado pelas mulheres, ocasionando assim uma diminuição na satisfação sexual, e a presença de sintomas depressivos interfere negativamente no funcionamento sexual normal (DANESH M, 2015).

Segundo Lonnée-Hoffmann R e Pinas I (2014), em um estudo cuja metodologia era uma revisão de literatura, obtiveram que apenas 10 a $20 \%$ das mulheres podem apresentar alguma disfunção sexual, principalmente nos domínios de dor e orgasmo, e a presença de sintomas depressivos pós-operatória é um fator preexistente e deve ser melhor informado as mulheres. A função dos órgãos envolvidos na excitação e no prazer sexual podem interferir de forma desfavorável na obtenção de satisfação em ambos os parceiros. O medo de sentir dor também pode estar presente na mulher, provocando uma atitude esquiva em relação ao sexo (MELO MCB e BARROS EM, 2009).

\section{CONCLUSÃO}

Conforme os dados dessa pesquisa, a histerectomia total pode prejudicar a função sexual de mulheres histerectomizadas tendo correlação com sintomas depressivos devido ao impacto que a cirurgia causa nos aspectos físicos e biopsicossociais. Foram encontrados na literatura metodologias e variáveis diferentes das utilizadas nesse estudo, há estudos que também relatam que houve uma melhora na função sexual e não houve correlação com sintomas depressivos pós histerectomia. No entanto, estes variam de acordo com as diferentes metodologias e variáveis utilizadas, portanto é necessária a realização de mais pesquisas com uma amostra maior e utilizando os mesmos questionários que foram utilizados nessa pesquisa. 


\section{REFERÊNCIAS}

1. ADORNO SS, et al. Vantagens da histerectomia vaginal comparado à histerectomia abdominal em mulheres sem prolapso genital. Revista Eletrônica Acervo Saúde,2018; (17): e97.

2. AARTS JWM, et al. Surgical approach to hysterectomy for benign gynaecological disease. Cochrane Database of Systematic Reviews, 2015; 8: 1-178.

3. ARAÚJO TVB, AQUINO, E. M. L. Fatores de risco para histerectomia em mulheres brasileiras. Cad. Saúde Pública, 2003; 19(Sup. 2):S407-S417.

4. BAHRI N, et al. Depressão após histerectomia e os fatores de influência. Iran Red Crescent Med J., 2016; 18(1): e30493.

5. BECK AT, et al. Psychometric properties of the Beck Depression Inventory: Twenty-five years of evaluation. Clinical Psychology Review, 1988; 8(1): 77-100.

6. CARDOSO BC, et al. Perfil de Mulheres Submetidas a Histerectomia e Influência da Deambulação na Alta Hospitalar. Pleiade, 2017; 11(21): 17-24.

7. CARVALHO HCM, LEMOS, MF. As Consequências Da Histerectomia Na Sexualidade Feminina. Perspectivas em Psicologia, 2017; 21(1): 209 -224.

8. COELHO SM. Perfil epidemiológico e complicações pós-operatórias das mulheres submetidas à cirurgia ginecológica em centro de referência do extremo setentrional da Amazônia legal brasileira. Rev. Col. Bras. Cir., 2015; 42(6): 372376.

9. COSTA JR; COSTA A. Tipos e vias de abordagem cirúrgica em histerectomia e sua relação com lesão do sistema urinário. Acta Obstet Ginecol Port. 2017; 11(1): 46-56.

10. DANESH M. O efeito da histerectomia na função sexual da mulher: uma revisão narrativa. Med Arch., 2015; 69(6): 387-92.

11. GHIELMETTI T, et al. Gynaecological operations: do they improve sexual life? Eur J Obstet Gynecol Reprod Biol., 2006; 129(2):104-110.

12. HARNOD T, et al. Hysterectomies Are Associated with an Increased Risk of Depression: A Population-Based Cohort Study. J Clin Med., 2018; 7(10): 366.

13. HENTSCHEL H, et al. Validação do female sexual function index (fsfi) para uso em língua portuguesa. Rev. HCPA, 2007; 27(1): 10-14.

14. ILLIANO E, et al. Hysterectomy and sexuality. First Published, 2016.

15. Kendall PC, et al. Issues and recommendations regarding use of the Beck Depression Inventory. Cogn Ther Res., 1987; 11(3): 289-299.

16. LOMMĖE-HOFFMANN R, PINAS I. Effects of Hysterectomy on Sexual Function. Curr Sex Health Rep., 2014; 6(4): 244-251.

17. LUNELLI BP, et al. O impacto da histerectomia abdominal no desempenho/ satisfação sexual. Arq Catarin Med., 2014; 43(1): 49-53.

18. MELO MCB, BARROS EN. Histerectomia E Simbolismo Do Útero: Possíveis Repercussões Na Sexualidade Feminina. Rev. SBPH, 2009; 12(2): 493-498.

19. MERIGHI MAB, et al. Experiências e expectativas de mulheres submetidas à histerectomia. Texto Contexto Enferm, Florianópolis, 2012; 21(3): 608-15.

20. NUNES MPRS, et al. Representações De Mulheres Acerca Da Histerectomia Em Seu Processo De Viver. Esc Anna Nery Rev Enferm., 2009; 13(3): 574-581.

21. PACAGNELLA RC, et al. Adaptação transcultural do Female Sexual Function Index. Cad. Saúde Pública [online], 2008; 24(2): 416-426.

22. PIASSAROLLI VP, et al. Treinamento dos músculos do assoalho pélvico nas disfunções sexuais femininas. Rev Bras Ginecol Obstet., 2010; 32(5): 234-240.

23. REAL AA, et al. Os efeitos da histerectomia sobre a sexualidade feminina. Saúde (Santa Maria), 2012; 38(2): $123-130$.

24. SANTOS JLC, et al. Função Sexual e Qualidade de Vida de Mulheres Submetidas à Histerectomia. Rev. Mult. Psic., 2017; 11(39): 1-11.

25. SILVA CMC, et al. A repercussão da histerectomia na vida de mulheres em idade reprodutiva. Esc Anna Nery Rev Enferm., 2010; 14(1): 76-82.

26. TOZO IM, et al. Avaliação da sexualidade em mulheres submetidas à histerectomia para tratamento do leiomioma uterino. Rev. Bras. Ginecol. Obstet., 2009; 31(10): 503-507.

27. WIEGEL M. Índice de função sexual feminina (FSFI): validação cruzada e desenvolvimento de pontos de corte clínicos. J Sex Marital Ther., 2005; 31(1): 1-20. 